\author{
M. J. A. Kamps \\ J. Horn \\ M. Oddo \\ J. E. Fugate \\ C. Storm \\ T. Cronberg \\ O. Wu \\ J. M. Binnekade \\ C. W. E. Hoedemaekers
}

\section{Response to De Jonghe et al.: Prognostication of neurological outcome after cardiac arrest: standardization of neurological examination conditions is needed}

Accepted: 22 November 2013

Published online: 5 December 2013

(C) Springer-Verlag Berlin Heidelberg and ESICM 2013

Dear Editor,

We thank Dr. De Jonghe for his interest in our meta-analysis on prognostication after cardiac arrest [1]. We agree with De Jonghe et al. that, in general, standardization of prognostic tests will improve the sensitivity and specificity of the test [2]. Specifically in patients after cardiac arrest treated with hypothermia, timing of the prognostic tests, dosage of the sedatives, timing and decision to withdraw active treatment, and a large number of other known and unknown confounders will influence the outcome. As this was a metaanalysis, we could not control the timing or technical performance of the diagnostic tests in the original observational studies. We believe that the post cardiac arrest treatment and prognostication protocols described in the original studies reflect current practice in a large number of hospitals worldwide. As such, the main conclusion of our analysis that absence of motor response and corneal reflexes at least $72 \mathrm{~h}$ after CPR are not reliable to predict poor outcome is robust and applicable to a large number of patients, despite the presence of possible confounders.

Conflicts of interest The authors have no conflict of interest.

\section{References}

1. Kamps MJ, Horn J, Oddo M, Fugate JE, Storm C, Cronberg T, Wijman CA, Wu O, Binnekade JM, Hoedemaekers CW (2013) Prognostication of neurologic outcome in cardiac arrest patients after mild therapeutic hypothermia: a metaanalysis of the current literature. Intensive Care Med 39:1671-1682. doi: 10.1007/s00134-013-3004-y

2. De Jonghe B, Sharshar T, Outin H (2014) Prognostication of neurologic outcome after cardiac arrest: standardization of neurological examination conditions is needed. Intensive Care Med. doi: 10.1007/s00134-013-3157-8

M. J. A. Kamps ·

C. W. E. Hoedemaekers (

Department of Intensive Care, Radboud

University Nijmegen Medical Centre, Geert Grooteplein zuid 10, 6525 GA Nijmegen, The Netherlands e-mail: C.Hoedemaekers@ic.umcn.nl

Tel.: +31-24-3617273

Fax: +31-24-3541612
M. J. A. Kamps

e-mail: marlijn.kamps@gmail.com

J. Horn · J. M. Binnekade

Department of Intensive Care, Academic Medical Center, Meibergdreef 9,

1105 AZ Amsterdam, The Netherlands e-mail: j.horn@amc.uva.nl

M. Oddo

Department of Intensive Care Medicine, Faculty of Biology and Medicine, LausanneUniversity Hospital, BH-08, Rue du Bugnon46, CHUV, 1011 Lausanne, Switzerland

e-mail: Mauro.Oddo@chuv.ch

\section{J. E. Fugate}

Department of Neurology, 200 First

St.S.W., Rochester, MN 55905, USA

e-mail: Fugate.Jennifer@mayo.edu

\section{Storm}

Deparment of Intensive Care Medicine/Nephrology, Charité

UniversitätsmedizinBerlin, Augustenburger Platz 1, 13353 Berlin, Germany e-mail: christian.storm@charite.de

\section{T. Cronberg}

Divisionof Neurology, Department of Clinical Sciences, Skåne University

Hospital,Lund University, 22363 Lund, Sweden

e-mail: tobias.cronberg@skane.se

$\mathrm{O} . \mathrm{Wu}$

Department of Radiology, Athinoula AMartinos Center for Biomedical Imaging, 149 13th Street, CNY 2301, Charlestown, MA 22363, USA

e-mail: ona@nmr.mgh.harvard.edu 